\title{
A CHARACTERISATION OF SEMISIMPLE CLASSES
}

\author{
by A. D. SANDS \\ (Received 23rd May 1979)
}

Throughout this paper we shall work in the class of associative rings. In (4) it was shown that a class of rings is a semisimple class if and only if it is closed under extensions and ideals and is coinductive. This establishes a duality between radical classes and semisimple classes. This result has been proved also for classes of alternative rings in (2). In the original work by Kuros (1) on this subject two conditions were used for semisimple classes, one of which was weaker than the assumption that the class is closed under ideals. This condition is that every non-zero ideal of a ring in the class should have a non-zero homomorphic image in the class. It is natural to ask whether in the above set of conditions the condition of being closed under ideals can be replaced by this weaker condition. This question is raised in (3) and in (5) but it is suggested there that, in order to compensate, the coinductive condition be replaced by the stronger condition that the class is closed under subdirect sums. In fact we shall show that the weaker condition may be used without needing to replace the coinductive condition. We also give examples to show independence relations among these conditions.

For future reference we list the following properties of non-empty classes of rings.

(A) A class $\mathscr{C}$ of rings is closed under extensions if whenever a ring $A$ contains an ideal $B$ such that $B \in \mathscr{C}, A / B \in \mathscr{C}$ then $A \in \mathscr{C}$.

(B) A class $\mathscr{C}$ of rings is coinductive if whenever a ring $A$ contains a descending chain of ideals $B_{i}$ such that $\bigcap B_{i}=0$ and $A / B_{i} \in \mathscr{C}$, for each $i$, then $A \in \mathscr{C}$.

(C) A class $\mathscr{C}$ of rings is closed under subdirect sums if whenever a ring $A$ contains a family of ideals $B_{i}$ such that $\bigcap B_{i}=0$ and $A / B_{i} \in \mathscr{C}$, for each $i$, then $A \in \mathscr{C}$.

(D) A class $\mathscr{C}$ of rings is closed under ideals if whenever $B$ is an ideal of a ring $A$ and $A \in \mathscr{C}$ then $B \in \mathscr{C}$.

(E) A class $\mathscr{C}$ of rings is regular if $0 \in \mathscr{C}$ and whenever $B$ is a non-zero ideal of a ring $A$ in $\mathscr{C}$ then $B$ has a non-zero image in $\mathscr{C}$.

It is clear that (D) implies (E). The class $\mathscr{C}$ consisting of $0, \mathbb{Z}$ and the fields $\mathbb{Z} / p \mathbb{Z}$, where $p$ takes all prime values, satisfies (E) but not (D).

It is clear that $(\underline{C})$ implies $(\underline{B})$. The class $\mathscr{b}$ consisting of 0 and a single field $\mathbb{Z} / p \mathbb{Z}$ satisfies (B) but not (C). It also satisfies (D), (E).

In (3), (5), the question is raised as to whether conditions $(\underline{A}),(\underline{C})$ and $(E)$ imply that a class is semisimple. In (4), Theorem 1 , it was shown that $(\underline{A}),(\underline{B})$ and $(D)$ imply that a class is semisimple. We shall show that $(\underline{A}),(B)$ and $(E)$ imply that a class is semisimple, which gives also a positive answer to this question of (3), (5). 
Theorem. Let a class $\mathscr{C}$ of rings satisfy (A), (B) and (E). Then $\mathscr{C}$ is a semisimple class.

Proof. In view of (4), Theorem 1, it suffices to show that $\mathscr{C}$ satisfies (D). Let $R$ be in $\mathscr{C}$ and let $A$ be a non-zero ideal of $R$. Let $B_{i}$ be the family of all ideals of $A$ such that $A / B_{i} \in \mathscr{C}$. By (E) $A$ contains at least one proper ideal with this property. From (B) and Zorn's Lemma it follows that this family contains minimal members. Let $B$ be such a minimal member. Then $A / B \in \mathscr{C}$ and if $K$ is an ideal of $A$ such that $K$ is strictly contained in $B$ it follows that $A / K \notin \mathscr{C}$. If $B=0$ then $A \in \mathscr{C}$, as required. So we may assume that $B \neq 0$. If $B$ is an ideal of $R$ then, by $(E), B$ has a non-zero image in $\mathscr{C}$. If $B$ is not an ideal of $R$ then there exists $r \in R$ such that either $r B \not \subset B$ or $B r \not \subset B$. We may suppose that $r B \not B$. Consider the mapping $\lambda_{r}: B \rightarrow A / B$ defined by $\lambda_{r}(b)=r b+B$ for all $b \in B$. It is routine to check that $\lambda_{r}$ is a ring homomorphism and that $\lambda_{r}(B)=$ $(r B+B) / B$ is an ideal of $A / B$. Since $(r B+B) / B$ is non-zero and $A / B \in \mathscr{C}$ it follows from $(E)$ that $\lambda_{r}(B)$, and hence $B$, has a non-zero image in $\mathscr{C}$. Thus in all cases, with $B \neq 0, B$ has a non-zero image in $\mathscr{C}$.

As above there is an ideal $C$ minimal in the family of ideals $C_{i}$ of $B$ such that $B / C_{i} \in \mathscr{C}$. If $C$ is an ideal of $A$ then $A / B \in \mathscr{C}, B / C \in \mathscr{C}$ implies, by (A), that $A / C \in \mathscr{C}$. This contradicts the minimality of $B$. Thus there must exist $a \in A$ such that either $a C \not \subset C$ or $C a \not \subset C$. We may assume $a C \not \subset C$. Again this gives rise to a ring homomorphism $\lambda_{a}: C \rightarrow B / C$, defined by $\lambda_{a}(c)=a c+C$, whose image $(a C+C) / C$ is a non-zero ideal of $B / C$. Since $B / C \in \mathscr{C}$ it follows by (E) that $(a C+C) / C$ has a non-zero image in $\mathscr{C}$. Let $D / C$ be minimal in the family of ideals $D_{i} / C$ of $(a C+C) / C$ such that $((a C+C) / C) /\left(D_{i} / C\right) \in \mathscr{C}$. Then $(a C+C) / D \in \mathscr{C}$. From $B(a C+C) \subset C$ it follows that $B D \subset D$. If $D B \not \subset D$ then there exists $b \in B$ with $D b \notin D$. As above there is a ring homomorphism $\rho_{\mathrm{b}}: D / C \rightarrow(a C+C) / D$ defined by $\rho_{\mathrm{b}}(d+C)=d b+D$ whose image $(D b+D) / D$ is a non-zero ideal of $(a C+C) / D$. From (E) it follows that $\rho_{b}(D / C)$, and hence $D / C$, has a non-zero image in $\mathscr{C}$. Let this image be $D / E$ where $C \subset E \varsubsetneqq D$ and $E$ is an ideal of $D$. From $(a C+C)^{2} \subset B(a C+C) \subset C$ it follows that $E$ is an ideal of $a C+C$. From $(\underline{A})$ it folows that $(a C+C) / E \in \mathscr{E}$, which contradicts the minimality of $D / C$. Therefore $D B \subset D$ and $D$ is an ideal of $B$. Let $\pi$ be the natural projection from $(a C+C) / C$ to $(a C+C) / D$. Let $K$ be the kernel of $\pi \circ \lambda_{a}$, i.e. $K=\{x \in C \mid a x \in D\}$. Then $C / K \cong(a C+C) / D$ and so $C / K \in \mathscr{C}$. Let $y \in B$; then, for each $x \in K$, axy $\in D y \subset D B \subset D$ and ayx $\in B x \subset B C \subset D$. It follows that $K$ is an ideal of $B$. From (A) and $B / C \in \mathscr{C}$, $C / K \in \mathscr{C}$ it follows that $B / K \in \mathscr{C}$. This contradicts the minimality of $C$.

Therefore $A$ belongs to $\mathscr{C}$. Thus $\mathscr{C}$ satisfies (D) and so is a semisimple class.

Corollary. If a class $\mathscr{C}$ of rings satisfies $(\underline{\mathrm{A}}),(\underline{\mathrm{C}})$ and $(\underline{\mathrm{E}})$ then $\mathscr{C}$ is a semisimple class.

Since every semisimple class satisfies the conditions $(\underline{A}),(\underline{B}),(\underline{C}),(\underline{D})$ and $(\underline{E})$ we have shown, indirectly, that $(\underline{A}),(\underline{B})$ and $(E)$ imply $(\underline{C})$. It is, perhaps, worth pointing out that no two of these three conditions are sufficient to imply $(C)$. We have already given an example of a class satisfying (B), (D) and (E) but not (C). There are many classes, such as the class of finite rings, which satisfy $(\mathrm{A}),(\mathrm{D})$ and $(\mathrm{E})$ but not $(\mathrm{C})$. To exhibit a class $\mathscr{C}$ of rings satisfying (A), (B) but not (C) we use the construction from (4). Let $\mathscr{C}_{1}$ be any class of rings and construct the following classes inductively: 
$\mathscr{C}_{\mu+1}=\left\{R \mid R\right.$ contains an ideal $A$ with $A$ and $R / A$ in $\left.\mathscr{C}_{\mu}\right\}$, for each ordinal $\mu$;

$\mathscr{C}_{\lambda}=\left\{R \mid R\right.$ contains a descending chain of ideals $A_{i}$ such that $\cap A_{i}=0$ and $R / A_{i} \in \mathscr{C}_{\mu_{i}}$ for ordinals $\left.\mu_{i}<\lambda\right\}$, for each limit ordinal $\lambda$.

Finally let $\mathscr{C}_{u}=\bigcup \mathscr{C}_{\mu}$. As in the proof of Theorem 2 of (4) it may be shown that $\mathscr{C}_{u}$ satisfies (A), (B). Now let $\mathscr{C}_{1}$ be the class of all rings of order $p^{2}$, where $p$ is a fixed prime. It is clear that new finite rings are constructed at stage $\mathscr{C}_{\mu}$ only when $\mu$ is a finite ordinal. Thus each finite ring in $\mathscr{C}_{u}$ has order $p^{2 m}$, for some integer $m$, and so no ring of order $p^{3}$ belongs to $\mathscr{C}_{u}$. However $\mathbb{Z} / p^{2} \mathbb{Z} \oplus \mathbb{Z} / p \mathbb{Z}$ is clearly a subdirect sum of rings of order $p^{2}$. Thus $\mathscr{C}_{u}$ is not closed under subdirect sums.

The construction given in (4) can also be used to obtain the least semi-simple class containing a regular class $\mu_{1}$. It is easy to check that the class $\mu_{\mu}$ remains regular at each stage of the construction. Thus the class $\mathcal{M}_{u}$ is regular and so is a class satisfying (A), (B) and (E) and thus, by the Theorem, is semi-simple. However if the class $\mu_{1}$ is not regular an analogous construction cannot be used since there need not be a least regular class containing $\boldsymbol{M}_{1}$. For example if $\mathscr{C}$ is the class consisting of $0, \mathbb{Z}$ and the fields $\mathbb{Z} / p \mathbb{Z}$ where $p \equiv 1(4)$ and $\mathscr{D}$ is the class consisting of $0, \mathbb{Z}$ and the fields $\mathbb{Z} / p \mathbb{Z}$ where $p \equiv 3(4)$ then, as above, $\mathscr{C}$ and $\mathscr{D}$ are regular classes, but $\mathscr{C} \cap \mathscr{D}=\{0, \mathbb{Z}\}$ is not regular. So $\mu_{1}=\{0, \mathbb{Z}\}$ is not contained in a least regular class.

\section{REFERENCES}

(1) A. G. Kuros, Radicals of rings and algebras, Mat. Sb. 33, (1953), 13-26 (Russian) (English Translation: Rings, Modules and Radicals, Colloq. Math. Soc. Janos Bolyai, 6 (1973), 297-314).

(2) L. C. A. Van Leeuwen, C. Roos and R. Wiegandt, Characterizations of semi-simple classes, J. Austral. Math. Soc. (Series A) 23 (1977), 172-182.

(3) L. C. A. VAN LeEUWEN and R. WIEgandt, Radicals, semisimple classes and torsion theories, Acta. Math. Acad. Sci. Hungar. (to appear).

(4) A. D. SAnds, Strong upper radicals, Quart. J. Math. (Oxford) (2) 27 (1976), 21-24.

(5) R. Wregandt, List of Problems-Kolloquium über Algebra, Vienna, (1978), 6.

Department of Mathematics

The UnIVERSITY

DundeE DD1 4HN 